\title{
Early intervention in panic: pragmatic randomised controlled trial
}

Peter Meulenbeek, Godelief Willemse, Filip Smit, Anton van Balkom, Philip Spinhoven and Pim Cuijpers

\section{Background}

Many people suffer from subthreshold and mild panic disorder and are at risk of developing more severe panic disorder.

\section{Aims \\ This study (trial registration: ISRCTN33407455) was conducted to evaluate the effectiveness of an early group intervention based on cognitive-behavioural principles to reduce panic disorder symptomatology.}

\section{Method}

Participants with subthreshold or mild panic disorder were recruited from the general population and randomised to the intervention $(n=109)$ or a waiting-list control group $(n=108)$. The course was offered by 17 community mental health centres.

\section{Results}

In the early intervention group, 43/109 (39\%) participants presented with a clinically significant change on the Panic Disorder Severity Scale-Self Report (PDSS-SR) v. 17/108 (16\%) in the control group (odds ratio (OR) for favourable treatment response $3.49,95 \% \mathrm{Cl} 1.77-6.88, P=0.001$ ). The course also had a positive effect on DSM-IV panic disorder status $(\mathrm{OR}=1.96,95 \% \mathrm{Cl}=1.05-3.66, P=0.037)$. The PDSS-SR symptom reduction was also substantial (between-group standardised mean difference of 0.68). The effects were maintained at 6-month follow-up.

\section{Conclusions}

People presenting with subthreshold and mild panic disorder benefit from this brief intervention.

\section{Declaration of interest}

None.
Early intervention in subthreshold or mild panic disorder is of public interest because it affects a sizeable population segment, ${ }^{1,2}$ is associated with a large burden of disease and generates considerable economic costs to society. ${ }^{3-5}$ Only a few studies have been conducted in early intervention for panic symptoms in adults. ${ }^{6,7}$ The results of these studies suggest that early intervention is a promising option. However, the research lacks generalisability, given the use of a limited sample (i.e. college students and people with panic attacks seen in an emergency room). The aim of this study (trial registration: ISRCTN33407455) was to examine the effectiveness of an early group intervention for panic symptoms, based on cognitive-behavioural therapy, and offered by community mental health centres, in a sample of self-referred people presenting with subthreshold or mild panic disorder. To strengthen the trial's external validity the present intervention was studied in its natural setting. We hypothesised that the intervention would show superior effects in reducing panic disorder symptomatology, relative to a waiting-list control group with unrestricted access to usual care.

\section{Method}

\section{Design}

We conducted a pragmatic, multisite, randomised controlled trial of the 'Don't Panic' course $v$. a waiting-list control group. Measurements were taken at baseline $\left(T_{0}\right)$, and at post-test after 3 months $\left(T_{1}\right)$. To monitor effect maintenance over time the early intervention group received an extended follow-up at 9 months after baseline, i.e. 6 months after the end of the course $\left(T_{2}\right)$. The randomisation took place after administration of the Mini International Neuropsychiatric Interview-Plus (MINI-Plus) ${ }^{8}$ and was carried out centrally by an independent third party. A blocked randomisation scheme was used, stratified by mental health centre, subthreshold panic disorder $v$. mild panic disorder, and by presence $v$. absence of co-occurring agoraphobia. The latter was included because it was assumed that agoraphobia is a prognostically relevant factor for treatment response in panic disorder. This procedure ensured that participants with and without panic disorder or agoraphobia were equally distributed across both trial arms. Power calculations indicated that 129 participants per group were required in order to detect a difference in symptom reduction, equivalent to a standardised effect size of at least 0.35 in a two-sided test at alpha $=0.05$ and a power of $(1$-beta $)=0.80$. The trial protocol was approved by an independent medical ethics committee (METIGG) and was conducted between September 2005 and July 2007.

\section{Participants}

Participants were recruited from the general adult population in The Netherlands. They were eligible if over 18 years of age and presenting with subthreshold or mild panic disorder, defined as having symptoms of panic disorder falling below the cut-off of 13 on the Panic Disorder Severity Scale-Self Report (PDSS-SR). ${ }^{9}$ Exclusion criteria were: severe panic disorder (PDSS-SR >12), current psychological treatment for panic disorder-related complaints, presence of other severe mental or social problems, suicidal intention warranting treatment or likely to interfere with participation in the group course as assessed by an experienced psychologist during intake. People meeting one of the exclusion criteria were advised to seek regular treatment. If a participant used medication for anxiety or depression (e.g. benzodiazepines or antidepressants) it was agreed to keep medication use stable during the study period. Eligible participants received a complete description of the study and only entered the trial once informed consent was obtained.

Participants were recruited according to the standard procedure for recruiting participants for preventive services in 
The Netherlands. All community mental health centres in The Netherlands have a prevention department, offering preventive and early interventions to the general population in their region. Many of these interventions are aimed at people with subthreshold disorders, and participants are recruited through media announcements, flyers in libraries and healthcare buildings, and via banners placed on the internet. In the current study, this was precisely the recruitment strategy used to recruit participants. There were 17 participating community mental health centres. For screening, the standard procedures employed by the community mental health centres were used. First, people who showed interest received more information about the course and the study. They also had an initial screening interview by telephone to ascertain the presence of panic symptoms. Second, potential participants had an interview with an experienced psychologist from a community mental health centre. In this interview, the inclusion and exclusion criteria as described earlier were checked. In addition, potential participants were interviewed by trained interviewers from the Trimbos Institute (Netherlands Institute of Mental Health and Addiction) using the MINI-Plus ${ }^{8,10}$ for research purposes. This was done to assess the DSM-IV ${ }^{11}$ panic disorder status as well as the possible presence of concurrent agoraphobia, and to exclude people presenting with severe depressive disorder.

\section{Interventions}

We developed an early intervention for panic symptoms, called the 'Don't Panic' course. The course was based on cognitivebehavioural principles that have been shown to be effective in the treatment of the full-blown disorder. ${ }^{12-14}$ This course was developed specifically for adults. It consisted of 8 weekly sessions of $2 \mathrm{~h}$ each in groups of 6-12 participants. The 'Don't Panic' course manual was used by the psychologist and prevention worker offering the intervention and there was an accompanying workbook for the participants. ${ }^{15}$ To ensure the integrity of intervention delivery, the course instructors were trained in offering the course and working with the course manual by the Trimbos Institute (i.e. a 1-day training programme by P.M.). In addition, they had access to a help desk when questions arose while offering the intervention. Participants were taught to examine their panic attacks and the possible causes, to use techniques to control anxiety levels and to develop coping skills. The course included:

(a) psychoeducation on the psychological and physiological nature of anxiety and panic attacks;

(b) lifestyle changes to improve their physical condition;

(c) stress management to prevent constant tension by learning effective ways to cope with stress;

(d) relaxation training to reduce physiological arousal;

(e) cognitive restructuring to challenge and correct dysfunctional cognitions about panic and anxiety;

(f) interoceptive exposure to reduce the fear of somatic sensations;

(g) 'in vivo' exposure to reduce agoraphobic avoidance and safety behaviours; and

(h) techniques aimed at relapse prevention.

Participants evaluated their progress during the course. Three months after completion of the course a booster session was offered to the participants. Each session was structured and encompasses a review of homework assignments, feedback, rehearsals, information about the upcoming topics and practical skills training. The course was extensively pilot tested before entering the clinical trial stage. ${ }^{16}$

The control group were put on a waiting list. These individuals were free to make use of other interventions for panic disorder. Therefore the control group could also be described as a care as usual group - with one difference: individuals in the waiting list group knew that they could start the course 1 month after the intervention group had completed the intervention.

\section{Measures}

We used the PDSS-SR and the MINI-Plus as the primary outcome measures. Severity of panic symptoms was measured with the PDSS-SR. ${ }^{17,18}$ The PDSS-SR generates a total score ranging from 0 to 28 , with a higher score indicating more severe panic symptoms. A cut-off score of 13 discriminates between mild and severe panic disorder. ${ }^{9}$ To assess the DSM-IV panic disorder and agoraphobia status the MINI-Plus ${ }^{8,10}$ was used. To exclude severe major depressive disorder the depression section of the MINI-Plus was supplemented with the Sheehan Disability Scale. ${ }^{19}$ Individuals presenting with severe impairments in at least two areas of role functioning because of a depressive disorder were excluded from the study. The interviews were conducted by trained interviewers who received 1 day's training from the Trimbos Institute (P.M. and G.W.). For efficiency, the interviews were conducted by telephone; an approach that can be justified from a psychometric point of view. ${ }^{20,21}$ The interviewers were masked to the randomisation status of the participants. The following self-rated questionnaires were used as secondary outcome measures. For agoraphobic avoidance the Mobility Inventory $^{22,23}$ was employed. The subscale for anxiety of the Hospital Anxiety and Depression Scale (HADS-Anxiety) ${ }^{24,25}$ was used to indicate anxiety levels. The Beck Depression Inventory $(\mathrm{BDI}-\mathrm{II})^{26,27}$ was used to assess depressive symptoms. All outcome measures have good psychometric properties. The self-report questionnaires were utilised for all three measurements and completed at home. The MINI-Plus was conducted by telephone at $T_{0}$ and $T_{1}$.

\section{Analyses}

As the primary outcome we compared the proportion of participants manifesting with a clinically significant change on the PDSS-SR across both groups. A clinically significant change was defined according to the criteria proposed by Jacobson \& Truax: ${ }^{28}$ a change should move from a dysfunctional distribution to a functional one, and the change should be statistically reliable in the sense that the observed change cannot be attributed to measurement error. As we studied a population with subthreshold and mild panic disorder, we considered scores below one standard deviation of the mean pre-test score on the PDSS-SR as falling within the functional range. ${ }^{29}$ Participants meeting the Jacobson \& Truax criterion were coded 1 (implying favourable treatment response, 'success') or else 0 ('failure'). This binary outcome was used to obtain the odds ratio (OR) using the logistic regression model and the number needed to treat (NNT) using a linear probability model for a binomial outcome (i.e. a generalised linear model for a binomial distributed error-term and using identity as the link function).

We tried to obtain converging evidence for the central clinical outcome by also evaluating the effect on panic disorder status using the MINI/DSM-IV criteria. As indicated, the sample can be divided into two groups: people with relatively mild manifestations of MINI/DSM-IV panic disorder $(n=100)$ and those with subthreshold manifestations not meeting the diagnostic criteria $(n=117)$. The latter group is said to be 'at risk' of 
developing panic disorder. In this group we observed how many remained panic disorder-free at $T_{1}$ (a favourable preventative outcome, 'success'). However, when attention is paid to the former group presenting with mild panic disorder a favourable treatment response occurs when a participant is panic disorder free at $T_{1}$ ('success'). This allows a comparison of the proportion of successes across both trial groups. Again, this yields a binomial outcome with failure coded as 0 , and success as 1 . In a next step, this binary outcome was used to obtain the OR and NNT as before.

For outcomes on continuous measurement scales, such as the PDSS and the HADS, a Gaussian regression model was used to test the hypothesis of superior effects in the early intervention arm as compared with the waiting-list control group. We also calculated standardised between-group mean differences (Cohen's $d$ effect size).

To test effect maintenance up to the extended follow-up at 9 months after baseline in the early intervention group, we used a paired-sample $t$-test to analyse the difference in mean score of the self-report measures in the early intervention group from $T_{0}$ to $T_{1}, T_{0}$ to $T_{2}$ and $T_{1}$ to $T_{2}$.

All analyses were conducted in agreement with the intentionto-treat principle, ${ }^{30}$ hence all participants were analysed in the group to which they were randomised, and missing end-points at follow-up were imputed using a regression model with the best available predictors of outcome and the best predictors for drop out. The first set of predictors is required to obtain the most precise estimates for the missing values; the latter set of predictors is used to correct for bias that may stem from differential loss to follow-up associated with $T_{0}$ variables. ${ }^{31}$

In all analyses we accounted for the clustering of data induced by the multisite character of the study. Clustering violates the assumption of independence of observations, and may thus affect standard errors and $P$-values. So-called 'robust standard errors' and correct $P$-values were obtained using the first-order Taylorseries linearisation method. All analyses were conducted with Stata 9.2 for Windows. All tests were conducted using a two-sided significance level of $P=0.05$.

\section{Results}

\section{Characteristics of the participants}

A total of 586 people expressed an interest in the course. During a first screening by telephone it transpired that 210 of them had psychiatric symptoms other than panic related ones (e.g. related to social phobia or generalised anxiety) or there were practical obstacles (work, travel distance) that precluded their participation in the trial. The remaining 376 participants were interviewed by an experienced psychologist. A total of 217 participants entered the study and were randomised to the early intervention group $(n=109)$ or the control group $(n=108)$ (Fig. 1). The baseline characteristics of the study participants are presented in Table 1.

In the sample, $71 \%$ were female. The mean age was 42 years, ranging from 20 to 75 years. Most participants were employed and

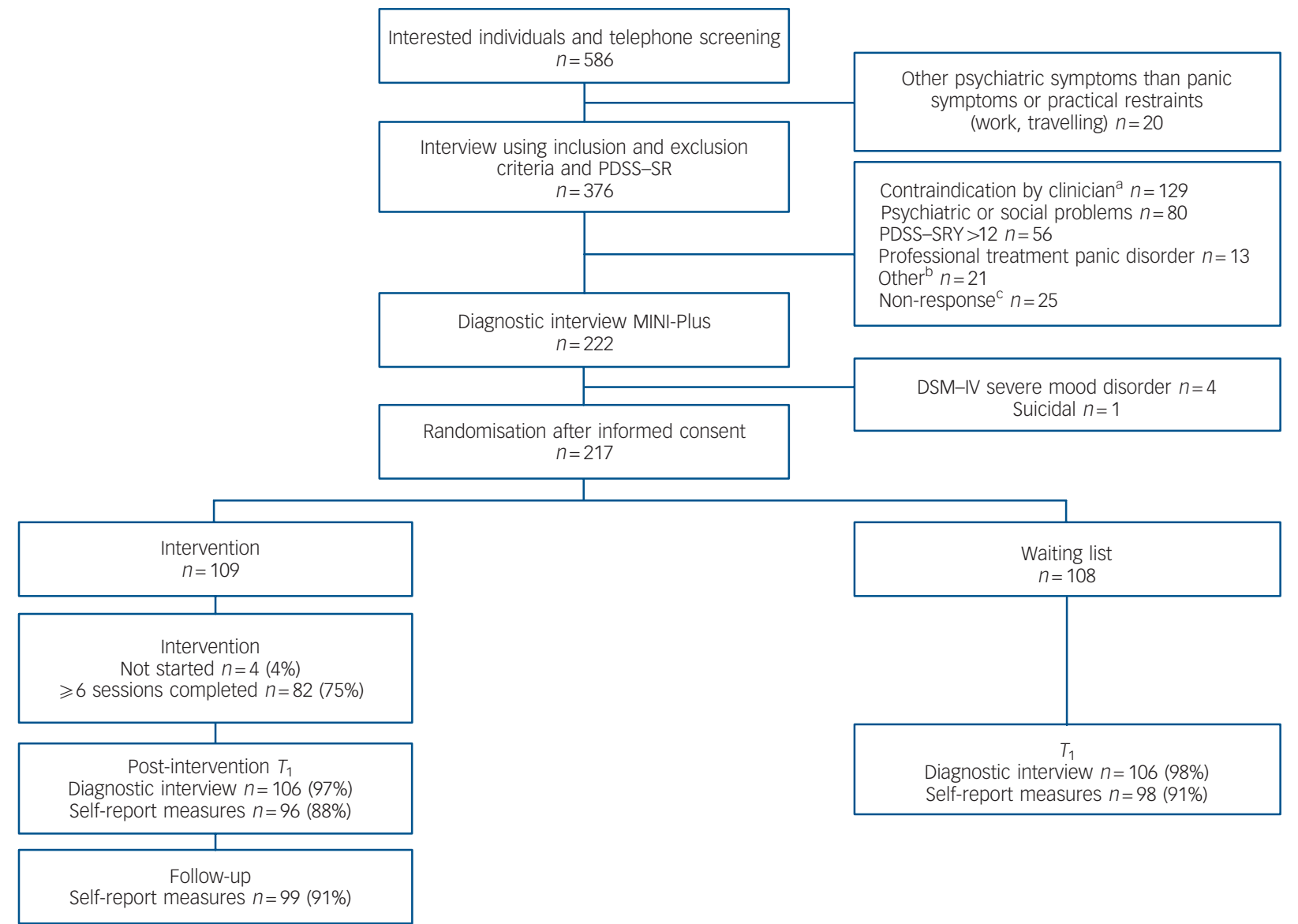

Fig. 1 Participants' flow through the study.

PDSS-SR, Panic Disorder Severity Scale-Self Report; MINI-Plus, Mini-International Neuropsychiatric Interview-Plus.

a. Participants can have more than one contraindication.

b. Including somatic problems $(n=11)$.

c. Including practical restraints. 


\begin{tabular}{|c|c|c|c|}
\hline & $\begin{array}{l}\text { Total group } \\
\qquad(n=217)\end{array}$ & $\begin{array}{l}\text { Early intervention group } \\
\qquad(n=109)\end{array}$ & $\begin{array}{l}\text { Control group } \\
\qquad(n=108)\end{array}$ \\
\hline Female, $n(\%)$ & $154(71)$ & $77(71)$ & $77(71)$ \\
\hline Age, years: mean (s.d.) range & 42 (12.4) 20-75 & 42 (12.9) 20-75 & $42(11.8) 20-74$ \\
\hline Married/living with partner, $n$ (\%) & $169(78)$ & $83(76)$ & $86(80)$ \\
\hline Employed (paid), $n$ (\%) & $151(70)$ & 75 (69) & $76(70)$ \\
\hline Years of education, mean (s.d.) & $14.04(3.26)$ & $14.07(3.29)$ & $14.01(3.25)$ \\
\hline MINI-Plus-Panic disorder: current, $n$ (\%) & $100(46)$ & $50(46)$ & $50(46)$ \\
\hline MINI-Plus-Agoraphobia: current, $n$ (\%) & $135(62)$ & $68(62)$ & $67(62)$ \\
\hline PDSS-SR, mean (s.d.) range & 7.18 (3.23) 0-28 & 7.02 (3.24) 0-28 & 7.35 (3.24) 0-28 \\
\hline HADS-Anxiety, mean (s.d.) range & $9.54(3.83) 0-21$ & 9.48 (3.94) 0-21 & $9.60(3.74) 0-21$ \\
\hline Mobility Inventory, mean (s.d.) range & $1.96(0.66) 1-5$ & $1.89(0.62) 1-5$ & $2.02(0.69) 1-5$ \\
\hline BDI-II, mean (s.d.) range & $12.46(7.64) 0-63$ & $11.96(8.01) 0-63$ & $12.97(7.26) 0-63$ \\
\hline
\end{tabular}

were living with a partner (Table 1). The early intervention and the control group did not differ significantly with regard to sociodemographic and clinical characteristics. Almost 50\% met the DSM-IV criteria of (mild) panic disorder at entry to the study, whereas 62\% were diagnosed with agoraphobia. Overall, 194 (89\%) participants completed the $T_{1}$ self-report questionnaire, with no significant difference in response rate between both groups. Completers did not differ significantly from non-completers on any of the baseline variables. Follow-up data for 99 participants ( $91 \%$ of the early intervention group) were available at 6 months after completion of the course.

\section{Clinically significant change}

The functional range turned out to be a score below 3.95 on the PDSS-SR; this is 4-scale points below the cut-off score of 8 that may discriminate between the presence or absence of current DSM-IV panic disorder. ${ }^{17,18}$ The reliable change on the PDSS-SR appeared to be a pre-post difference of at least 3.85 scale points. We coded for 'success' when a reliable pre-post change of 3.85 scale points had occurred and when, in addition, the cut-off value of 4 on the PDSS-SR was crossed. In the early intervention group, $43 / 109$ (39\%) participants $v .17 / 108$ (16\%) participants in the control group presented with a successful outcome: $\mathrm{OR}=3.49$, $95 \%$ CI $1.77-6.88, P=0.001, \mathrm{NNT}=4.2$ under an intention-totreat analysis.

It was further tested whether there was any difference in the primary outcome between people with subthreshold panic disorder and those with mild panic disorder. A logistic regression analysis revealed no significant difference $(\mathrm{OR}=0.88$; robust s.e. $=0.6755 ; t=-0.16, P=0.878)$.

\section{Effect on MINI/DSM-IV diagnostic status}

In the early intervention group, $89 / 109$ (82\%) participants presented with a favourable treatment response on the MINI-Plus compared with $75 / 108(69 \%)$ participants in the control group (Table 2; $\mathrm{OR}=1.96,95 \%$ CI 1.05-3.66, $P=0.037$, NNT $=8.2$ ).

\section{Effect on panic severity}

The course was found to have a beneficial effect on reducing panic severity levels compared with the waiting-list control group (PDSS-SR, $P=0.004$ ). The mean score on the PDSS-SR at $T_{1}$ for the early intervention group was $3.48 v .5 .77$ for the control group. The standardised effect size on the PDSS-SR at $T_{1}$ was 0.68 ; from a clinical perspective this can be interpreted as a large effect. $^{32}$

\section{Other outcomes}

The scores for the other outcomes are presented in Table 3. Undertaking the course was superior to being in the waiting-list control group on all outcomes. The between-group effect sizes for these secondary outcomes ranged from 0.31 to 0.59 .

There was no significant difference in the use of medication between the groups at baseline. In the early intervention group, 36 (33\%) participants used medication at baseline, $3(3 \%)$ started medication during the course and $9(8 \%)$ stopped using medication. In the control group, 48 (44\%) participants used medication at baseline, $7(6 \%)$ started and $8(7 \%)$ stopped medication in the period between baseline and $T_{1}$. Therefore, it is unlikely that the present findings can be explained by changes in medication use.

\section{Six-month follow-up}

The difference in mean scores on the self-report measures of the early intervention group from $T_{0}$ to $T_{1}$ and $T_{0}$ to $T_{2}$ were all significant at $P<0.001$. The mean score at $T_{2}$ on the PDSS-SR $($ mean $=3.48$, s.d. $=3.42)$ and the HADS-Anxiety (mean 6.13,

\begin{tabular}{|c|c|c|c|c|}
\hline \multirow[b]{2}{*}{ Panic disorder-status } & \multicolumn{2}{|c|}{ Early Intervention group $(n=109)$} & \multicolumn{2}{|c|}{ Control group $(n=108)$} \\
\hline & $n$ & $\%$ & $n$ & $\%$ \\
\hline Subthreshold panic disorder at $T_{0}$ & 59 & & 58 & \\
\hline Success (stayed panic disorder-free at $T_{1}$ ) & 51 & 86 & 43 & 74 \\
\hline Mild panic disorder at $T_{0}$ & 50 & & 50 & \\
\hline Success (became panic disorder-free at $T_{1}$ ) & 38 & 76 & 32 & 64 \\
\hline Total success on the MINI-Plus & 89 & 82 & 75 & 69 \\
\hline
\end{tabular}




\begin{tabular}{|c|c|c|c|c|}
\hline & $\begin{array}{l}\text { Early intervention } \\
\qquad(n=109)\end{array}$ & $\begin{array}{l}\text { Control } \\
(n=108)\end{array}$ & Effect size ${ }^{a}$ & $P^{\mathrm{b}}$ \\
\hline \multicolumn{5}{|c|}{ Panic Disorder Severity Scale-Self Report (range 0-28) } \\
\hline$T_{0}$, mean (s.d.) & $7.02(3.24)$ & $7.35(3.24)$ & & \\
\hline$T_{1}$, mean (s.d.) & $3.48(3.22)$ & 5.77 (3.54) & 0.68 & 0.004 \\
\hline \multicolumn{5}{|c|}{ Hospital Anxiety and Depression Scale, subscale Anxiety (range 0-21) } \\
\hline$T_{0}$, mean (s.d.) & $9.48(3.94)$ & $9.60(3.74)$ & & \\
\hline$T_{1}$, mean (s.d.) & $6.26(3.93)$ & $8.61(4.00)$ & 0.59 & 0.001 \\
\hline \multicolumn{5}{|c|}{ Mobility Inventory (range 1-5) } \\
\hline$T_{0}$, mean (s.d.) & $1.89(0.62)$ & $2.02(0.69)$ & & \\
\hline$T_{1}$, mean (s.d.) & $1.67(0.57)$ & $1.95(0.70)$ & 0.44 & 0.004 \\
\hline \multicolumn{5}{|c|}{ Beck Depression Inventory-Second edition (range 0-63) } \\
\hline$T_{0}$, mean (s.d.) & $11.96(8.01)$ & $12.97(7.26)$ & & \\
\hline$T_{1}$, mean (s.d.) & $9.20(7.89)$ & $11.58(7.69)$ & 0.31 & 0.036 \\
\hline
\end{tabular}

s.d. $=3.81)$ of the early intervention group did not differ significantly from $T_{1}$ (PDSS-SR: $P=0.995$; HADS-Anxiety: $P=0.627)$. The mean score at $T_{2}$ on the Mobility Inventory $($ mean $=1.61$, s.d. $=0.60)$ and the BDI-II (mean 7.83, s.d. =7.53) were significantly lower than at $T_{1}$ (Mobility Inventory: $P=0.037$; BDI-II: $P=0.007)$. The results suggest that the improvement after the course was maintained at 6-month follow-up.

\section{Acceptability}

After each session the attendance of the participants was registered and it was ascertained whether they had carried out their homework assignments. On the basis of this information it can be concluded that four participants in the early intervention group (4\%) did not start the course. Reasons for not starting were beginning another course and lack of time because of work. Eighty-two (75\%) participants in the early intervention group completed the course (completing the course is defined as attending at least six sessions). The main reasons for not completing the course were practical obstacles (work, illness). The mean number of attended sessions was 6.3 sessions. Of the attending participants, $83 \%$ had completed their homework for each session, $15 \%$ did not complete their homework for one session, $1.4 \%$ for two sessions, $0.5 \%$ for three sessions and $0.5 \%$ for four sessions. Therefore, compliance with the course was satisfactory.

The post-test self-report questionnaire of the early intervention group provided information about satisfaction with the course (response rate $83 \%, n=91$ ). The participants evaluated the course positively (organisational aspects, coaching, content, group sessions and workbook). Asked whether the course had contributed to being better able to manage panic complaints, $88 \%$ answered in the affirmative. The participants rated psychoeducation about anxiety and panic attacks, changing lifestyle, relaxation training and cognitive restructuring as most helpful. On a scale ranging from 1 'very bad' to 10 'excellent', the mean rating for the course was 7.8 (range: $5-9$, s.d. $=0.945$ ). These findings add to the impression that the course is acceptable.

\section{Discussion}

\section{Main findings}

We hypothesised that the 'Don't Panic' course would be superior in reducing panic disorder symptomatology. The results show that the participants in the early intervention group made significantly more improvement on panic symptoms compared with the waiting-list control group on all outcomes. Furthermore, symptoms of depression declined, as measured by the BDI-II. This finding is important because people with panic symptoms may also have higher rates of depressive symptoms. ${ }^{33}$ To our knowledge this is the first study that shows the effectiveness of an early group intervention for self-referred adults with subthreshold or mild panic disorder. The improvements were maintained over 6 months after the course. These findings are in agreement with previous findings on prevention and early intervention in panic disorder. ${ }^{6,7}$ The difference between the present sample and clinical samples ${ }^{17,18}$ is emphasised by the substantially lower mean degree of severity of panic symptoms as measured with the PDSS-SR at baseline. To explore the effectiveness of the intervention in other samples, future research might also use clinical samples, particularly in primary care settings. The finding that there was no difference in the primary outcome between people with subthreshold panic disorder and those with mild panic disorder could have implications for general clinical practice because it seems to indicate that an early intervention in less severe panic could perhaps best be implemented broadly as a first step in a stepped care mental health approach. People with panic symptoms will vary in the type and intensity of interventions they prefer. To increase the coverage of the target population, exploring alternative ways of intervening such as collaborative care and guided self-help may address that need.

\section{Acceptability of the course}

The participants who evaluated the course assessed it as positive and helpful. Compliance with the course was satisfactory. These findings suggest the course is suitable and acceptable. This is furthermore supported by the fact that many (about 40\%) community mental health centres in The Netherlands now offer the course on a regular basis. In addition, a group intervention may be more cost-effective than an individual intervention. An economic evaluation of the intervention for a subgroup of the sample (i.e. people with subthreshold panic disorder) showed that it may be acceptable from a cost-effectiveness point of view. ${ }^{34}$ The results may be highly generalisable, as the course was examined in its natural setting and the recruitment strategy of the study and the community mental health centres that offer the course are comparable. Another strength of this study was the high response rate. 


\section{Limitations}

We recognise a number of limitations in this study. First, because of the absence of a placebo control it is not clear whether nonspecific components of the course such as social interaction and expectation of gain contribute to the early intervention effect. Future research should use placebo-controlled designs to overcome this limitation. Second, the period allocated to studying change in the panic disorder status was only 3 months. The control group received the intervention a few weeks after $T_{1}$. In future research, longer follow-up is recommended to study changes in the incidence of panic disorder. Third, the extended follow-up in the treatment group was only 6 months following the conclusion of the course. Longer follow-up periods are needed to find out how long the beneficial effects will persist.

\section{Implications}

Overall, our findings carry the promise that prevention and early intervention through a brief group intervention for people with subthreshold or mild panic disorder can be effective. The selected target group is known to be reticent in asking professional help and it is therefore good to see that a low-threshold intervention is apparently regarded as accessible and acceptable.

Peter Meulenbeek, MSC, Department of Clinical Psychology and EMGO Institute,
VU-University, Amsterdam, and GGNet, Community Mental Health Centre, Warnsveld;
Godelief Willemse, PhD, Trimbos Institute (Netherlands Institute of Mental Health
and Addiction), Utrecht; Filip Smit, PhD, Institute of Extra-Mural Medicine, VU
University Medical Centre, Amsterdam, and Trimbos Institute (Netherlands Institute
of Mental Health and Addiction), Utrecht; Anton van Balkom, PhD, Department
of Psychiatry and EMGO Institute, VU-University Medical Centre, Amsterdam;
Philip Spinhoven, PhD, Institute of Psychology and Department of Psychiatry, Leiden
University, Leiden; Pim Cuijpers, PhD, Department of Clinical Psychology and EMGO
Institute, VU-University, Amsterdam, The Netherlands

Correspondence: Peter Meulenbeek, GGNet, PO Box 2003, 7230 GC Warnsveld, The Netherlands. Email: p.meulenbeek@compaqnet.nl

First received 28 Aug 2009, final revision 4 Dec 2009, accepted 5 Jan 2010

\section{Funding}

Supported by The Netherlands Health Research Council, The Hague (ZonMw) grant number 50-50110-98-035 (awarded to F.S.).

\section{Acknowledgements}

The authors thank Ms Jessica Herzmanatus, MSC, (GGNet) and Ms Rianne van der Zanden MSc, (Trimbos Institute) for their help with the development of the 'Don't Panic' course; and the trainers and trainees for their valuable assistance in making this study possible.

\section{References}

1 Norton GR, Cox BJ, Malan J. Nonclinical panickers: a critical review. Clin Psychol Rev 1992; 12: 121-39.

2 Batelaan N, De Graaf R, Van Balkom A, Vollebergh W, Beekman A. Thresholds for health and thresholds for illness: panic disorder versus subthreshold panic disorder. Psychol Med 2007; 37: 247-56.

3 Harvison K, Woodruff-Borden J, Jeffery S. Mismanagement of panic disorder in emergency departments: contributors, costs, and implications for integrated models of care. J Clin Psychol Med Settings 2004; 11: 217-32.

4 Marciniak M, Lage M, Landbloom R, Dunayevich E, Bowman L. Medical and productivity costs of anxiety disorders: case control study. Depress Anxiety 2004; 19: 112-20.

5 Smit F, Cuijpers P, Oostenbrink J, Batelaan N, De Graaf R, Beekman A. Costs of nine common mental disorders: implications for curative and preventive psychiatry. J Ment Health Policy Econ 2006; 9: 193-200.

6 Gardenswartz CA, Craske MG. Prevention of panic disorder. Behav Ther 2001; 32: 725-37.

7 Swinson RP, Soulios C, Cox BJ, Kuch K. Brief treatment of emergency room patients with panic attacks. Am J Psychiatry 1992; 149: 944-6.

8 Sheehan DV, Lecrubier $\mathrm{Y}$, Sheehan $\mathrm{KH}$, Amorim $\mathrm{P}$, Janavs J, Weiller $\mathrm{E}$, et al. The Mini International Neuropsychiatric Interview (MINI): the development and validation of structured diagnostic psychiatric interview for DSM-IV and ICD-10. J Clin Psychiatry 1998; 59 (suppl 20): 22-33.
9 Michelson D, Allgulander C, Dantendorfer K, Knezevic A, Maierhofer D, Micev V, et al. Efficacy of usual antidepressant dosing regimens of fluoxetine in panic disorder. Randomised, placebo-controlled trial. Br J Psychiatry 2001; 179: 514-8.

10 Van Vliet IM, Leroy H, Van Megen HJGM. M.I.N.I. Plus: M.I.N.I. Internationaal Neuropsychiatrisch Interview: Dutch version 5.0.0. Leiden LUMC, 2000.

11 American Psychiatric Association. Diagnostic and Statistical Manual of Mental Disorders (4th edn) (DSM-IV). APA, 1994.

12 Bakker A. Recente ontwikkelingen in de behandeling van paniekstoornis en agorafobie [Recent developments in the treatment of panic disorder and agoraphobia]. Tijdschrift voor Psychiatrie 2001; 43: 385-93.

13 Margraf J, Barlow DH, Clark DM, Telch MJ. Psychological treatment of panic: works in progress on outcome, active ingredients, and follow-up. Behav Res Ther 1993; 31: 1-8.

14 Van Balkom AJLM, Bakker A, Spinhoven P, Blaauw BMJW, Smeenk S, Ruesink B. A meta-analysis of the treatment of panic disorder with or without agoraphobia: a comparison of psychopharmacological, cognitivebehavioral, and combination treatments. J Nerv Ment Dis 1997; 185: 510-6.

15 Meulenbeek P, Herzmanatus J, Smit F, Willemse G, Van der Zanden R. Draaiboek: Geen Paniek, Leren Omgaan met Paniekklachten [Manual and Workbook: Don't Panic, Learn to Cope with Panic Complaints]. Trimbos Institute/GGNet, 2005.

16 Meulenbeek P, Willemse G, Smit F, Smits N, Van Balkom A, Spinhoven P, et al. Effects and feasibility of a preventive intervention in sub-threshold and mild panic disorder: results of a pilot study. BMC Res Notes 2009, 2: 4.

17 Shear MK, Rucci P, Williams J, Frank E, Grochocinski V, Vander Bilt J, et al. Reliability and validity of the panic disorder severity scale: replication and extension. J Psychiatr Res 2001; 35: 293-6.

18 Van der Meer A, Burgerhout K. Nederlandse versie van de Panic Disorder Severity Scale [Dutch version of the Panic Disorder Severity Scale]. Universiteit van Amsterdam, 2004.

19 Leon AC, Olfson M, Portera L, Farber L, Sheehan DV. Assessing psychiatric impairment in primary care with the Sheehan Disability Scale. Int J Psychiatry Med 1997; 27: 93-105.

20 Rohde P, Lewinsohn PM, Seeley JR. Comparability of telephone and face-toface interviews in assessing axis I and II disorders. Am J Psychiatry 1997; 154: 1593-8.

21 Evans M, Kessler D, Lewis G, Peters TJ, Sharp D. Assessing mental health in primary care research using standardized scales: can it be carried out over the telephone? Psychol Med 2004; 34: 157-62.

22 Chambless DL, Caputo GC, Jasin SE, Gracely EJ, Williams C. The Mobility Inventory for agoraphobia. Behav Res Ther 1985; 23: 35-44.

23 De Beurs E. The Assessment and Treatment of Panic Disorder and Agoraphobia. Thesis Publications, 1993.

24 Zigmond AS, Sniath RP. The Hospital Anxiety and Depression Scale. Acta Psychiatr Scand 1983; 67: 361-70.

25 Spinhoven P, Ormel J, Sloekers PPA, Kempen GIJM, Speckens AEM, Van Hemert AM. A validation study of the Hospital Anxiety and Depression Scale (HADS) in different groups of Dutch subjects. Psychol Med 1997; 27: 363-70.

26 Beck AT, Steer RA, Brown GK. Manual for the Beck Depression Inventory-II. Psychological Corporation, 1996.

27 Van der Does AJW. Handleiding bij de Nederlandse versie van de Beck Depression Inventory, Second Edition (BDI-II-NL) [Manual of the Dutch version of the Beck Depression Inventory, Second Edition (BDI-II-NL)]. Swets Test Publishers, 2002.

28 Jacobson NS, Truax P. Clinical significance: a statistical approach to defining meaningful change in psychotherapy research. J Consult Clin Psychol 1991; 59: 12-9.

29 Greeven A, Van Balkom AJLM, Visser S, Merkelbach JW, Van Rood YR, Van Dyck $R$, et al. Cognitive behavior therapy and paroxetine in the treatment of hypochondriasis: a randomized controlled trial. Am J Psychiatry 2007; 164: 91-9.

30 Everitt BS. Modern Medical Statistics: A Practical Guide. Oxford University Press, 2003.

31 Demirtas $\mathrm{H}$. Simulation driven inferences for multiply imputed longitudinal datasets. Stat Neerl 2004; 58: 466-82.

32 Lipsey MW, Wilson DB. The efficacy of psychological, educational and behavioral treatment. Confirmation from meta-analysis. Am Psychol 1993; 48: 1181-209.

33 Birchall $\mathrm{H}$, Brandon S, Taub N. Panic in a general practice population: prevalence, psychiatric comorbidity and associated disability. Soc Psychiatry Psychiatr Epidemiol 2000; 35: 235-41.

34 Smit F, Willemse G, Meulenbeek P, Koopmanschap M, Van Balkom A, Spinhoven $P$, et al. Preventing panic disorder: cost-effectiveness analysis alongside a pragmatic randomised trial. Cost Eff Resour Alloc 2009; 7: 8. 